\title{
In vivo dual-modality imaging of lymphatic systems using indocyanine green in rats: three-dimensional photoacoustic imaging and planar fluorescence imaging
}

Chulhong Kim, Kwang Hyun Song, Lihong V. Wang

Chulhong Kim, Kwang Hyun Song, Lihong V. Wang, "In vivo dual-modality imaging of lymphatic systems using indocyanine green in rats: threedimensional photoacoustic imaging and planar fluorescence imaging," Proc. SPIE 7564, Photons Plus Ultrasound: Imaging and Sensing 2010, 75642W (23 February 2010); doi: 10.1117/12.841964

SPIE. Event: SPIE BiOS, 2010, San Francisco, California, United States 


\title{
In vivo dual-modality imaging of lymphatic systems using indocyanine green in rats: three-dimensional photoacoustic imaging and planar fluorescence imaging
}

\author{
Chulhong Kim ${ }^{1}$, Kwang Hyun Song ${ }^{2}$, and Lihong V. Wang ${ }^{1 *}$ \\ ${ }^{1}$ Optical Imaging Laboratory, Department of Biomedical Engineering, Washington University in St. \\ Louis, Campus Box 1097, One Brookings Dr. St. Louis, Missouri, 63130 \\ ${ }^{2}$ Division of Medical Physics \& Engineering, Department of Radiation Oncology, The University of \\ Texas Southwestern Medical Center at Dallas, 5323 Harry Hines Boulevard, Dallas, Texas 75390
}

\begin{abstract}
The purpose of this study is to map non-invasively sentinel lymph nodes (SLNs) and lymphatic vessels of rats in vivo using FDA-approved indocyanine green (ICG) and two non-ionizing imaging modalities: volumetric spectroscopic photoacoustic (PA) imaging, which measures optical absorption, and planar fluorescence imaging, which measures fluorescent emission. SLNs and lymphatic vessels were clearly visible after a $0.2 \mathrm{ml}$-intradermal-injection of $1 \mathrm{mM}$ ICG in both imaging systems. We also imaged deeply positioned lymph nodes in vivo by layering biological tissues on top of rats. These two modalities, when used together with ICG, have the potential to map SLNs in axillary staging and to study tumor metastasis in breast cancer patients.
\end{abstract}

Keywords: Photoacoustic tomography, planar fluorescence imaging, sentinel lymph node biopsy, breast cancer, axillary lymph node dissection.

\section{INTRODUCTION}

To minimize post-operative complications of axillary lymph node dissection (ALND), sentinel lymph node biopsy (SLNB), a biopsy of the first draining lymph node in breast cancer region, is the standard process for staging breast cancer patients. ${ }^{1}$ Although current SLNB using methylene blue dye and radioactive materials has an identification rate of $90-95 \%$ and a sensitivity of $88-95 \%$, this method is ionizing and invasive. Moreover, the false negative rate is still 5$10 \%$. ${ }^{2,3}$ In addition, due to the surgical removal of the sentinel lymph node, the SLNB still poses postoperative complications like ALND. ${ }^{4}$ As a minimally invasive method to reduce abovementioned complications, ultrasoundguided fine needle aspiration biopsy (FNAB) has been tested clinically. ${ }^{5}$ Yet, ultrasound imaging itself cannot distinguish the sentinel lymph node from other lymph nodes. Therefore, there is still a pressing need to develop an accurate, reliable, non-ionizing, and non-invasive detection method for lymph node assessment.

Photoacoustic (PA) imaging is an optical imaging modality that can provide strong optical contrast and high ultrasonic resolution. ${ }^{6,7}$ The image resolution can be tuned with ultrasonic frequency where diffusive photons reach. Moreover, the pixel number, defined as the ratio between the maximum imaging depth and the spatial resolution, maintains over 100. In biological tissues, the imaging depth can be up to a few centimeters deep. Methylene blue and gold nanoparticles have been applied as lymph node tracers in rats in vivo using PA imaging. ${ }^{8,9}$ However, the methylene blue dye can cause a chemical mastitis as well as photodamage under intense light. Furthermore, gold nanoparticles are not yet approved by the FDA for clinical applications.

We have used FDA-approved indocyanine green (ICG) dye for identifying SLNs and lymphatic vessels in PA imaging. Since the fluorescence quantum yield of ICG is about $10 \%$, it can also be used as a contrast agent for fluorescence imaging. ${ }^{10}$ Thus, the purpose of this study was to explore the feasibility of mapping in vivo SLNs and

\footnotetext{
* Corresponding author: 1hwang@biomed.wustl.edu
}

Photons Plus Ultrasound: Imaging and Sensing 2010, edited by Alexander A. Oraevsky, Lihong V. Wang, Proc. of SPIE Vol. 7564, 75642W · @ 2010 SPIE · CCC code: 1605-7422/10/\$18 · doi: 10.1117/12.841964 
lymphatic vessels with non-invasive and non-ionizing volumetric PA and planar fluorescence imaging, using FDAapproved ICG in rats.

\section{METHODS AND MATERIALS}

The experimental schematic of a PA imaging system is described in Fig 1a. ${ }^{11}$ A tunable dye laser (ND6000, Continuum) pumped by a Q-switched Nd:YAG laser (LS-2137, LOTIS) with 6-ns pulse duration and 10-Hz pulse repetition rate was utilized. A light wavelength of $668 \mathrm{~nm}$ was used for in vivo imaging. The ring-shaped light illumination was formed by a concave lens and a spherical conical lens. The light traveled down through an optical condenser, and was coaxially aligned with the ultrasound focus in water. ${ }^{12}$ The light fluence on the skin was $3.4 \mathrm{~mJ} / \mathrm{cm}^{2}$, within the current ANSI limit. ${ }^{13}$ We used a single-element 5-MHz ultrasound transducer (V308, Panametrics-NDT) to detect generated PA waves. The axial resolution is $144 \mu \mathrm{m}$ and the lateral resolution is $560 \mu \mathrm{m}$. Time-resolved PA amplitudes provide one dimensional depth-sensitive images (A-line). Mechanical raster scanning provides two dimensional depth-resolved (Bscan) and three dimensional images. The scanning stepsizes for the transverse directions (X and Y) were $200 \mu \mathrm{m}$ and $400 \mu \mathrm{m}$, respectively. For a $25 \mathrm{~mm}$ x $30 \mathrm{~mm}$ field of view, the acquisition time was 23 minutes.

The experimental schematic of a planar fluorescence imaging system is shown in Fig 1b. The light beam (Thorlabs, $785 \mathrm{~nm}, 80 \mathrm{~mW}$ ) was expanded by a concave lens and illuminated rats. A macro lens, a notch-plus filter, and a fluorescent bandpass filter were mounted onto the front of a cooled CCD camera (BU401-BR, Andor). Emitted fluorescent light was collected by the camera.

(a)
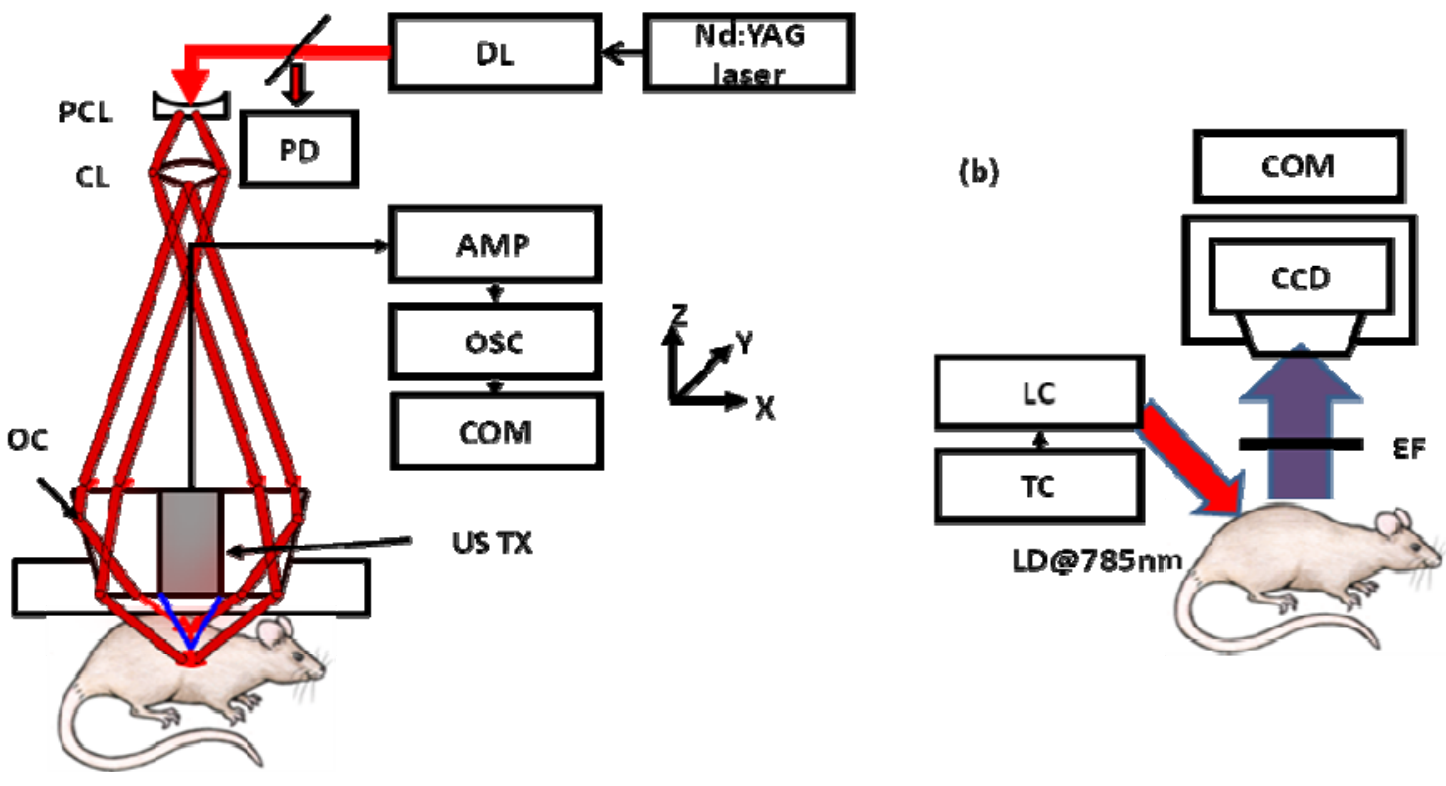

Figure 1: (a) The experimental schematic of volumetric photoacoustic imaging system. (b) The experimental setup of planar fluorescence imaging system. DL; dye laser, PCL; plano-concave lens, CL; conical lens, OC; optical condenser, US TX; ultrasound transducer, AMP; amplifier, OSC; oscilloscope, COM; computer, PD; photodiode, CCD; CCD camera, LC; laser controller, TC; temperature controller, LD; laser diode.

All animal experiments were performed according to the guidelines of the institution on the care and use of laboratory animals. A Sprague Dawley rat weighing $250-420 \mathrm{~g}$ was given a mixture of ketamine $(85 \mathrm{mg} / \mathrm{kg})$ and xylazine $(15 \mathrm{mg} / \mathrm{kg})$ to anesthetize him. After depilation in the axillary region, the rat was positioned atop a home-made holder. During the experiments, the rat was fully anesthetized by a vaporized-isoflurane system $(1 \mathrm{~L} / \mathrm{min}$ oxygen and $0.75 \%$ isoflurane, Euthanex), and the vitals were managed by a pulse oximeter. After image acquisition, the animal was euthanized with an overdose of pentobarbital. $0.2 \mathrm{ml}$ of 1-mM ICG was injected intradermally on the left forepaw pad of the rat. 


\section{RESULTS AND DISCUSSIONS}

To investigate the feasibility of the use of ICG for volumetric in vivo mapping of SLNs and lymphatic vessels, the left axilla of a rat was non-invasively imaged using the PA imaging system. Before the administration of ICG, a control image (MAP) was obtained, showing clear vascular structures with good contrast, $7.9 \pm 0.8$ (Fig 2a). A series of PA images was acquired after the injection of ICG. In Fig 2b, the SLN and lymphatic vessels, along with surrounding blood vessels, are clearly shown at $0.2 \mathrm{hr}$ post-injection. Figure $2 \mathrm{c}$ shows a B-scan image acquired along the cut in Fig. $2 \mathrm{~b}$. The SLN is positioned about $1.5 \mathrm{~mm}$ below the surface. In five PA imaging rats, SLNs were clearly visible with the PA signal enhancement of $5.9 \pm 1.8$ at $0.2 \mathrm{hr}$ post-injection, while lymphatic vessels were seen in four rats with the PA signal enhancement of $4.3 \pm 0.6$. We examined the degradation of spatial resolution in PA images when the imaging depth was incremented. After adding one $4 \mathrm{~mm}$ thick layer of chicken breast, the depth was increased to $5-6 \mathrm{~mm}$. In Fig. 2d, the SLN is clearly seen in the B-scan image. The spatial resolution is comparable with that in Fig. 2c.
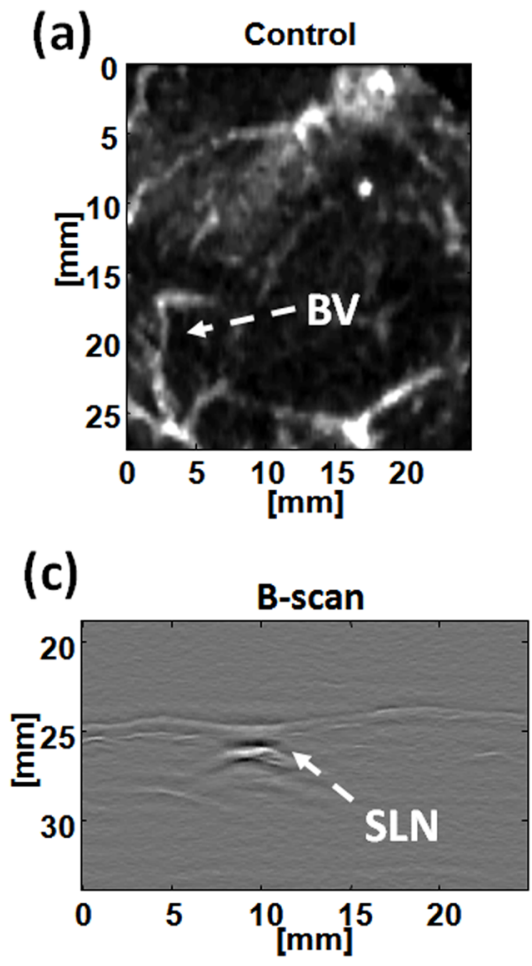
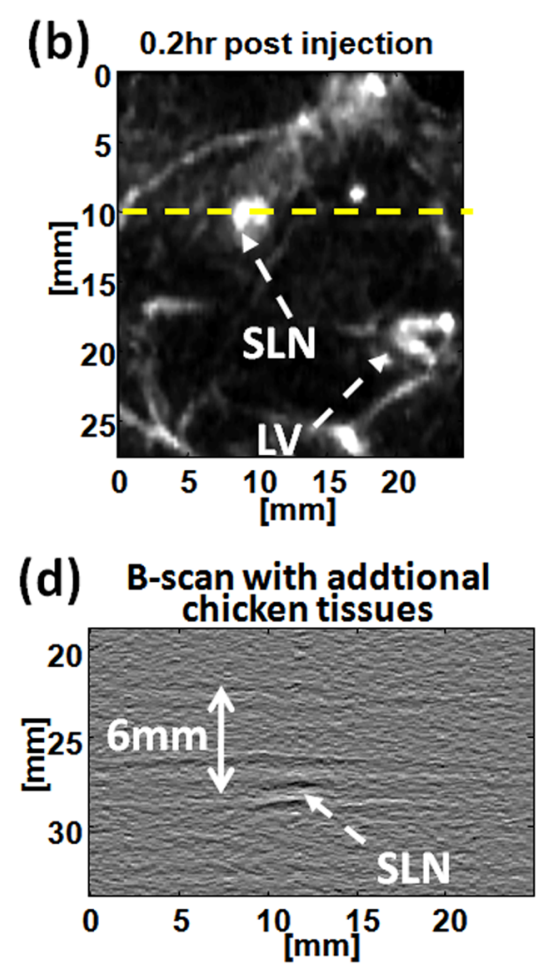

Figure 2: In vivo noninvasive PA images of lymphatic systems in rats. (a) Control MAP image acquired before ICG injection in the axillary region. (b) PA image at $0.2 \mathrm{hr}$ post-injection. (c) B-scan PA image along the cut in b. (d) B-scan PA image of a SLN with additional chicken tissues on top of the rat. BV; blood vessel, SLN; sentinel lymph node, and LV; lymphatic vessel.

By using the planar fluorescence imaging system, the dynamics of ICG uptake on the SLN were monitored in Fig. 3. Right after the injection, the SLN started showing up. As time passed, the fluorescent signal within the SLN became stronger, and reached a steady state 40 minutes after the injection. The SLN as well as lymphatic vessels are clearly shown, which is similar to the PA imaging results. Although planar fluorescence imaging is real-time, it does not provide any depth information in the images. The SNR and spatial resolution become worse when the imaging depth increases. 


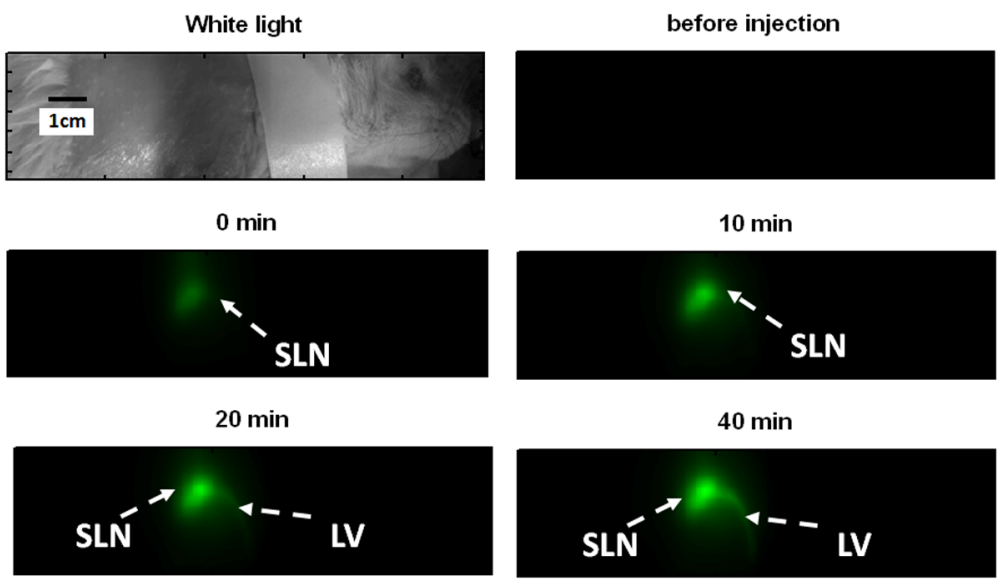

Figure 3: Planar fluorescence images acquired before injection (control) and 0 minutes, 10 minutes, 20 minutes, and 40 minutes postinjection of ICG. SLN; sentinel lymph node and LV; lymphatic vessel.

\section{CONCLUSIONS}

In conclusion, using volumetric spectroscopic PA and planar fluorescence imaging, we have demonstrated the feasibility of non-invasive in vivo dual-modality mapping of SLNs and lymphatic vessels with ICG. Unlike gold nanoparticles, ICG is an FDA-approved NIR dye for human use. Unlike methylene blue, it has less chemical side effect and photodamage. By applying a spectroscopic approach, this PA imaging system is potentially more useful in clinical conditions. Images from both techniques clearly show SLNs and lymphatic vessels (Figs 2 and 3). Due to the slow image acquisition (23 minutes for a $25 \mathrm{~mm} \times 30 \mathrm{~mm}$ field of view), this PA imaging system is not suitable to monitor the dynamics of ICG in SLNs and lymphatic vessels, compared to the fluorescence imaging. However, this is not a fundamental limitation of PA imaging. The imaging speed can be overcome if an ultrasound array ${ }^{14,15}$ is used as a detector, and/or a high laser pulse repetition rate with a high speed scanning system is used. In summary, non-invasive dual-modality (volumetric spectroscopic PA and planar fluorescence imaging) in vivo mapping of SLNs and lymphatic vessels using an FDA-approved ICG, has been successfully accomplished in rats. For clinical applications, further improvements are required, such as in portability and speed.

\section{ACKNOWLEDGEMENT}

This work was supported in part by grants from National Institutes of Health (R01 EB000712, R01 EB008085, R01 NS46214, and U54 CA136398 -- the Network for Translational Research -- to L.V.W.). L.V.W. has a financial interest in Endra, Inc., which, however, did not support this work.

\section{REFERENCES}

[1] H. Kobayashi, S. Kawamoto, Y. Sakai, et al., "Lymphatic drainage imaging of breast cancer in mice by micromagnetic resonance lymphangiography using a nano-size paramagnetic contrast agent," J. Natl. Cancer. I. 96(9), 703708 (2004).

[2] K. M. McMasters, T. M. Tuttle, D. J. Carlson, et al., "Sentinel lymph node biopsy for breast cancer: a suitable alternative to routine axillary dissection in multi-institutional practice when optimal technique is used," J. Clin. Oncol., 18(13), 2560-2566 (2000).

[3] O. A. Ung, "Australasian experience and trials in sentinel lymph node biopsy: the RACS SNAC trial," Asian. J. Surg. 27(4), 284-290 (2004).

[4] A. D. Purushotham, S. Upponi, M. B. Klevesath, et al., "Morbidity after sentinel lymph node biopsy in primary breast cancer: Results from a randomized controlled trial," J. Clin. Oncol. 23(19), 4312-4321 (2005). 
[5] S. Krishnamurthy, N. Sneige, D. G. Bedi, et al., "Role of ultrasound-guided fine-needle aspiration of indeterminate and suspicious axillary lymph nodes in the initial staging of breast carcinoma," Cancer 95(5), 982-988 (2002).

[6] X. Wang, Y. Pang, G. Ku, et al., "Noninvasive laser-induced photoacoustic tomography for structural and functional in vivo imaging of the brain," Nat. Biotechnol. 21(7), 803-806 (2004).

[7] H. F. Zhang, K. Maslov, G. Stoica, et al., "Functional photoacoustic microscopy for high-resolution and noninvasive in vivo imaging," Nat. Biotechnol. 24(7), 848-851 (2006).

[8] K. H. Song, E. W. Stein, J. A. Margenthaler, et al., "Noninvasive photoacoustic identification of sentinel lymph nodes containing methylene blue in vivo in a rat model," J. Biomed. Opt. 13(5), 054033 (2008).

[9] K. H. Song, C. Kim, C. M. Cobley, et al., "Near-Infrared Gold Nanocages as a New Class of Tracers for Photoacoustic Sentinel Lymph Node Mapping on a Rat Model,” Nano Lett. 9(1), 183-188 (2009).

[10] M. Li M, J. Oh, X. Xie, et al., "Simultaneous Molecular and Hypoxia Imaging of Brain Tumors In VivoUsing Spectroscopic Photoacoustic Tomography," Proceedings of IEEE 96(3), 481-488 (2008).

[11] K. H. Song and L. V. Wang, "Deep reflection-mode photoacoustic imaging of biological tissue," J. Biomed. Opt. 12(6), 060503 (2007).

[12] K. Maslov, G. Stoica, and L. V. Wang, "In vivo dark-field reflection-mode photoacoustic microscopy," Opt. Lett. 30(6), 625-627 (2005).

[13] Oregon Medical Laser Center Web site. http://omlc.ogi.edu/spectra/icg/index.html. Updated 2007. Accessed September 4, 2008.

[14] S. Liang, M. Konstantin, R. Bitton, et al., "Fast 3-D dark-field reflection-mode photoacoustic microscopy in vivo with a 30-MHz ultrasound linear array," J. Biomed. Opt. 13(5), 054208 (2008).

[15] P. Ephrat, M. Roumeliotis, F. S. Prato1, et al., "Four-dimensional photoacoustic imaging of moving targets," Opt. Express 16(26), 21570-21581 (2008). 